\title{
MAS for decentralized control of the Hybrid Electrical System consumption using fuzzy logic
}

\author{
Zaid BARI and Majid BEN YAKHLEF \\ Laboratory of Engineering Sciences (LSI) \\ Faculty Polydisciplinary Taza, USMBA, Fez, Morocco \\ zaid.bari@usmba.ac.ma \\ majid.benyakhlef@usmba.ac.ma
}

\begin{abstract}
The objective of this work is, firstly, to set up a model management of different element of a self-sufficient hybrid electrical system (HES) adapted the potentials (wind and solar) of isolated rural regions. Secondly to set up an energy management system able to establish an energy consumption policy taking account service requested by users, energy sources availability and loads various constraints. The aim of the management strategy is to adapt the energy demanded by the loads to that provided by renewable sources of production. It is based on the shift, interruption, reduction, or increase of the power requested from one or several controllable loads of the system. The suggested strategy, which is based on the flexibility of loads and used the fuzzy logic, has made it possible to reduce the lost energy that accounts for a significant gain from an economic point of view. It improves the use of the energy produced by modifying the charge profile. Proposed strategy privileges the functioning of the critical charges.
\end{abstract}

Keyword- Hybrid Electrical System, Multi Agent System, Contract net protocol, Fuzzy logic, Complex system.

\section{INTRODUCTION}

This document is a template. An electronic copy can be downloaded from the conference website. For questions on paper guidelines, please contact the conference publications committee as indicated on the conference website. Information about final paper submission is available from the conference website.

Before submitting your final paper, check that the format conforms to this template. Specifically, check the appearance of the title and author block, the appearance of section headings, document margins, column width, column spacing and other features.

Electrical energy management includes the protection, monitoring and control of the entire electrical network. For the operator, the issue is to optimize the energy consumption costs of different production sources without any prejudice to the activity. This is done through a smart, distributed and real time control of the whole set of parameters of the electrical system. The modern solutions to this control need consist in products and services using information and communication technologies, built around smart systems such as measurement stations and supervision and control software.

The application of wind and photovoltaic energy in electric power system is growing rapidly due to enhanced public concerns to adverse environmental impacts and escalation in energy costs associated with the use of conventional energy sources. Electric power from wind or photovoltaic energy is quite different from that of conventional resources. The fundamental difference is that the wind or solar power is intermittent and uncertain. Therefore, it affects the reliability of power system in a different manner from that of the conventional generators.

Research work on the distributed control of electrical systems holds an important part of the current bibliography [1-11]. This trend has allowed the development of several distributed control systems applied to microgrids. Several architectures and methods are presented with simulations showing their efficiencies in a microgrid control. The Multi-agent system (MAS) applied to the distributed control of electrical systems presents a major issue for the management of the complexity and flexibility of the control. Indeed, the loads (LD) and production source (PS) of an electrical network are multiple, of different types, geographically distributed and maintain several forms of interactions. The balance stresses between production and consumption, taking into account local problems (shutdown, etc.) and resource availability (sunshine, wind speed etc.), require realtime decentralized control and management system permanent adaptation. A MAS for the optimization of a decentralized multi-source system management was developed in our previous research work [12]. The proposed strategy has helped obtain a decentralized management of the energy production sources of the electrical system in the face of the shifts in the demand. The model proposed has allowed the global production to be optimized in relationship with the demand profile and in function of cost minimization criteria or even 
greenhouse gas reduction. In [13], the authors present a MAS designed for the real-time management of the functioning of a microgrid. The multi-agent system proposed provides a common communication interface for all the microgrid components. In [14-16], the authors present a design and an implementation method for the intelligent management of electrical distribution grids. These researches are of particular importance as they focus primarily on production while end favoring to adapt the production sources to the demand and trying to minimize the production cost and/or reduce the amount of the greenhouse gas emissions from the electrical system.

The mains questions asked in this work are:

Is the moment chosen by the user to ask for a given energy service, appropriate?

Is it possible to find a better moment to launch a given a service without compromising the user's comfort?

Is it possible to adapt the energy services requested in relation to the availability of the renewable energy sources?

The hybrid electrical system considered in this study includes two energy sources (photovoltaic, wind) accompanied by energy storage. This work suggests a management model based on the fuzzy logic and the coordination between MAS agents for decentralized self-efficient electrical network. The model presents an approach aiming at adapting consumption to production.

This work is organized as follows: Section 2 Present the methodological approach through models of the various components of the system, namely: model of the mathematical formulation of the control strategy of the electrical system, typology, model agents, protocol communication and coordination between agent. In the same section, the methodological approach adopted is detailed through behavioural modelling based on fuzzy logic and the coordination of multi-agent systems by contractual network protocol for a hybrid electric system (HES). Sections 3 present the analyses of the results obtained. We finished by conclusion.

\section{MATERIEL AND METHOD.}

The structure of the hybrid electrical system case study designed in the paper is shown in figure. 1, the small wind turbine, photovoltaic array and batteries are connected to the DC bus through the converter and the bus is connected to the loads through the inverter. The Aerogenerator and photovoltaic array mainly convert renewable energy sources such as wind energy and solar energy into electric energy; the batteries are used for energy regulation, and load balance. The total renewable power produced by the renewable energy sources is provided by Equation (1).

$$
\mathrm{P}_{\mathrm{ren}}(t)=\mathrm{P}_{\mathrm{pv}}(t)+\mathrm{P}_{\text {Aer }}(t)
$$

\section{A. The structure of the MAS-ECCS}

Four Agents are designed in the paper, including small wind turbine (WT) Agent, Photovoltaic (PV) Agent, Storage (Bat) Agent and Load (LD) Agent. Each Agent can control several generating unit of the same characteristics and determine its own control objective according to the characteristics of the equipment. The structure of MAS-ECCS (Multi Agent System energy coordination control strategy) is shown in figure. 2.

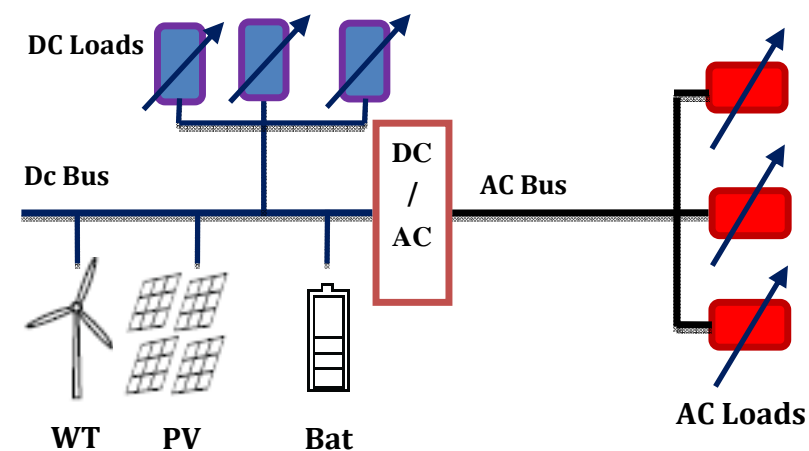

Fig 1. The Hybrid Electrical System studied (HES).

The system adopts the non-fixed client-server cooperative mechanism. Facing the conditions such as changes of the demand of energy and the meteorological data, the corresponding Agent should initiate the energy coordination task as the control center to ensure the equilibre between production and consumption. The Agent initiating the energy coordination task is considered to be Manager Agent (M). Other Agents determine how to participate in the energy coordination according to their own characters and control objectives. In order to avoid the conflict caused by several Agents initiating the energy coordination tasks at the same moment, the timestamp Task- Token is set to be the symbol of Task Agent. Only the Manager Agent with Task-Token in the effective period of time can initiate the energy coordination task. 
The FIPA97-based contract net protocol (Smith, 1980) is adopted between Agents to be the way of communication and the quad data $\angle \mathrm{B}, \mathrm{C}, \mathrm{S}>$ is designed to be the bidding data submitted by the participants to the initiators. Among them, B represents the bid value of the active output which can be a constant or a scope; $\mathrm{C}$ is the constraint condition, such as the maximum output power of the equipment, the capacity and service life of the batteries. $\mathrm{S}$ is the priority of each service to be connected or disconnected to the electrical system.

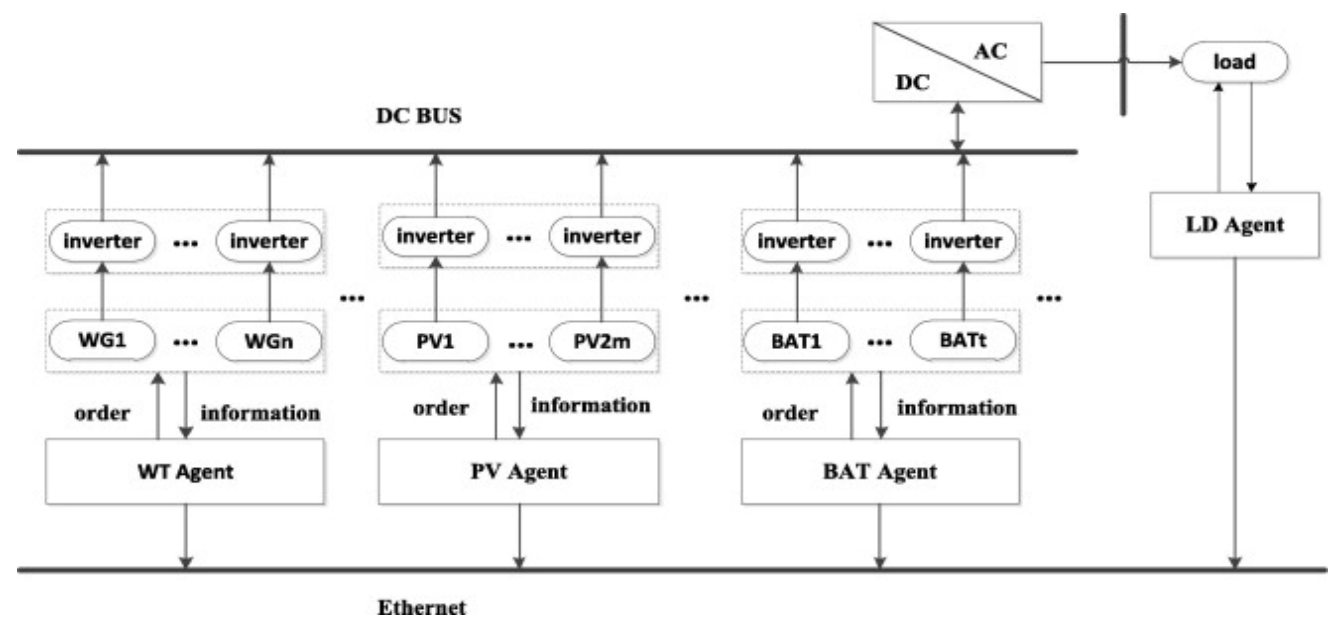

Fig 2. The structure of MAS-ECCS.

\section{B. Agent modeling and characteristic analysis}

The methodology adopted consists in transposing the energy flow control issue in an agent space where the system agent's entities cooperate smartly. To this end, each source is associated with a software agent. Each software agent implements the electrical (mathematical), behavior and source management model (of the source) it represents.

1) Output Power of PV Agent and WT Agent:

PV Agent and WT Agent represent the control units of the photovoltaic panel and the small wind turbine, respectively. As the photovoltaic panel and the small wind turbine operate at a low levelized cost, in the principle of maximizing the use of renewable energy, the characteristics of PV Agent and WT Agent are designed to be participating in the bidding actively until all the available equipment operate to the maximum power output. At the same time, they can initiate the energy coordination task when the power output changes.

The output power model of the PV Agent is determined by solar radiation and environ-mental temperature [17], as follows:

$$
P_{P V}(t)=A_{P V} G(t) \eta_{P V}(t) \eta_{i n v}
$$

Where $A_{P V}(\mathrm{~m} 2)$ is the area of the PV panel exposed to solar radiation, $G(t)(\mathrm{W} / \mathrm{m} 2)$ is the value of solar radiation, $\eta_{P V}(t)$ is the energy conversion efficiency of the PV module, and $\eta_{i n v}$ is the conversion efficiency of the inverter. $\eta_{P V}(t)$ is influenced by environmental temperature:

$$
\begin{aligned}
& \eta_{P V}(t)=\eta_{\text {ref }}\left[1-\beta\left(T_{c}(t)-T c r e f\right)\right] A_{P V} G(t) \eta_{\text {inv }} \\
& T_{c}(t)-T_{\text {ambiant }}=\frac{T_{\text {rated }}}{800} G(t)
\end{aligned}
$$

$\eta_{r e f}$ is the reference energy conversion efficiency of the PV module under a standard temperature, $\beta$ is the influence coefficient of the temperature for energy conversion efficiency, $T_{c}(t)$ is the temperature of the PV module at $\mathrm{t}$, and $T_{\text {cref }}$ is the reference standard temperature of the PV module, $T_{\text {ambiant }}$ is the ambient temperature, and $T_{\text {rated }}$ is the rated temperature of the PV module.

The output powers of the WT Agent in function of the wind speed as follows [18]. 


$$
P_{W T}(t)= \begin{cases}P n \frac{V^{k}(t)-V_{d}^{k}(t)}{V_{n}^{k}-V_{d}^{k}}, & V d<V(t)<V n ; \\ P n & V n<V(t)<V c ; \\ 0 & \text { Elswhere. }\end{cases}
$$

Where $V(t), V_{d}, V_{n}$ and $V_{c}$ respectively represent the instantaneous wind, start, nominal and cut off speed. $P n$ is the aerogenerator nominal power; $k$ is the form factor (without any dimension characterizing the Weibull distribution dissymmetry).

\section{2) Battery Agent:}

Energy storage system is necessary because the generation of renewable energy is inherently intermittent. The stored energy can supply the load when there is a lack of electricity, and store surplus power when the generated power exceeds the load. The state of charge at the moment $t$ can be obtained using the following equation 6 and 7 [19]:

Charge:

$$
\operatorname{SOC}(t)=\operatorname{SOC}(t-1)(1-\sigma)+\frac{P_{C h} \Delta t \eta_{C h}}{\mathrm{E}_{B a t}}
$$

Discharge:

$$
\operatorname{SOC}(t)=\operatorname{SOC}(t-1)(1-\sigma)+\frac{P_{D i s} \Delta t}{E_{B a t} \eta_{D i s}} \Delta \mathrm{t}
$$

Where $\operatorname{SOC}(t)$ is the battery state of charge after $\mathrm{t} ; \sigma$ is the self-discharge ; $P_{C h}$ and $P_{D \text { is }}$ are the charge and discharge powers of the battery in $t$, respectively ; $\Delta t$ is the length of $\mathrm{t}, \eta_{C h}$ and $\eta_{D i c}$ are the charge and discharge efficiencies of the battery, respectively and $\mathrm{E}_{\mathrm{Bat}}$ is the maximum capacity of the battery.

The battery state of charge must be kept in a reasonable range:

$$
\operatorname{SOC}_{\text {min }} \leq \operatorname{SOC}(t) \leq S O C_{\text {max }}
$$

Where $S O C_{\min }$ and $S O C_{\max }$ are minimum and maximum of battery capacity.

The decision making method of storage Agent is relatively simple. After receiving the bidding requirements of Manager Agent, it determines the charge and discharge interval [PCH_MAX (t), PDIS_MAX (t)] to be the bid value B according to Eqs. (6), (7) and (8) and accepts any valid value in the interval distributed by Manager Agent.

\section{3) LD Agent and Service Agent:}

The Load agents of the system are at an active or inactive state. A load agent is at the active state so long it consumes energy. It can be shifted, changed or interrupted depending on whether energy is available or not, of its priority and the electrical constraints. LD Agent can make the real-time monitoring of the change of the load. When the load demand changes, LD Agent can also apply for Manager Agent to initiate the energy coordination. It deserves noting that when LD Agent receives the bid invitation and becomes the participant, it will regard the current load demand and the priority of each service as the bid value B to send back. After the energy coordination by Manager Agent, if the returned bid value $\mathrm{R}$ is 0 , it suggests the balanced energy coordination; if the returned bid value $\mathrm{R}$ is greater than zero, it shows that the power generation unit cannot meet the load demand of the load. At this time, the LD Agent starts the process of adapting the load profile to production.

The agents representing the load are gathered depending on the services they provide.

A service is the transformation of energy by one or more loads to meet the needs of a user. Each service $S v_{k}$ is characterized by their degree of flexibility, which is centered on the demand shift, change or interruption:

- Demand shift: displacement of the service demand in a more favorable execution period without compromising the user's comfort.

- Service change: increasing or reducing the power requested by the service.

- Service interruption: consists in temporarily stopping the service and in starting it again at a more favorable period.

The Service Agents supervise locally the different groups of LD agents. They are associated with each distinct service. They monitor the agents of a same service by supervising the activities of the service groups and the determination process of the LD Agent priorities. The priority determination process is started when there is a conflict between LD Agents. 
A Service Agent implements the model of the fuzzy Logic Controller, shown in figure. 3 to determine the priority of each load $\mathrm{k}$ to be connected or disconnected from the network.

The frequency of execution of the service $\mathrm{k}$ noted $F_{S v k}$ with $(k=1,2, \ldots, n)$, in a time interval Ii (i indicates the index of the time interval I), is defined by an expert of system. The priority of the LD Agent $\mathrm{k}$ is a decisive parameter in the system control and makes it possible to activate or not the LDk Agents. It depends on the frequency of execution of the service $\mathrm{k}$ and the difference between the energy produced and the energy to be consumed by the loads, named $\varepsilon(\mathrm{t})$.

The Fuzzy Logic Controller FLC consists of $n$ parallel controllers; each controller has two inputs and one output. The two input variables of the FLCk are $\varepsilon(t)=\mathrm{P}_{\text {ren }}(\mathrm{t})-\mathrm{P}_{\text {load }}(t)$ and Frequency of service execution of each service $k(k=\{1,2, \ldots, n\})$ noted $F_{S V k}$. The output variable $S_{k}(\mathrm{t})$ represents the priority of each service to be executed (connected or disconnected to power system).The membership functions used in this study are triangular types in which the input/output relations are depicted in Fig 4.

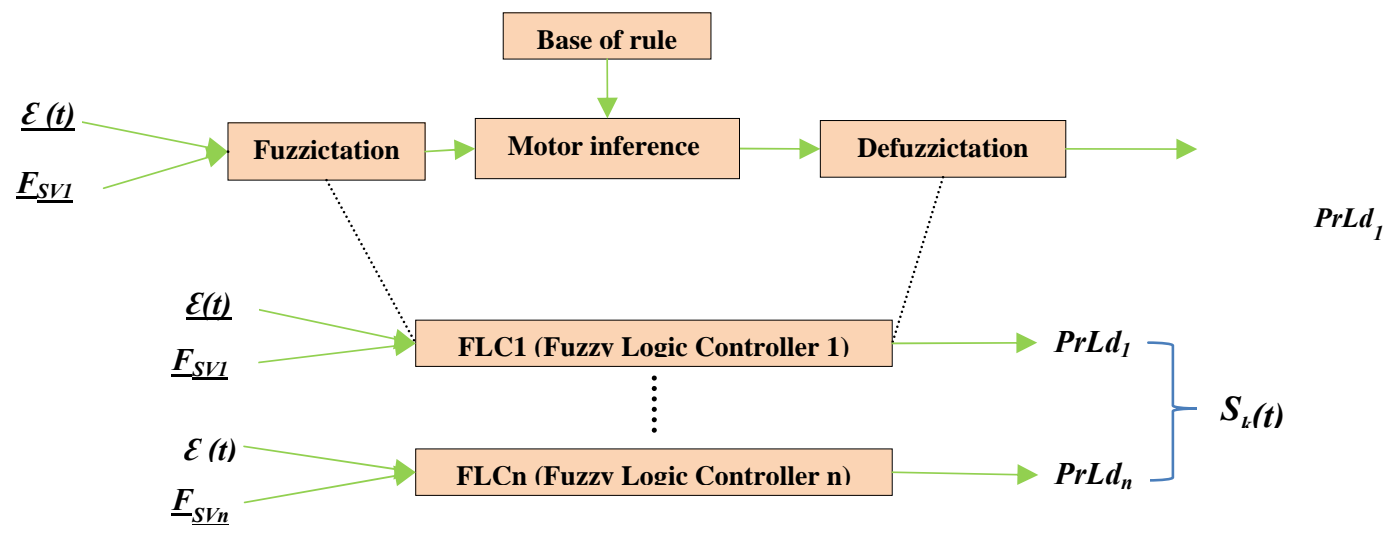

Fig 3. Architecture of the Fuzzy Logic Controller (FLC).

The first membership function linguist variables for a set of inputs $\varepsilon(t)$ are as following: Negative Verry Higher (NVH) Negative Higher (NH), Negative Medium (NM), Negative Lower (NL), Positive Lower (PL), Positive Medium (PM), Positive Higher (PH) and Positive Verry Higher (PVH). For the second input $F_{S V k}$ the membership function are: Verry Low Frequency (VLF), Low Frequency (LF), Medium Frequency (MF), Height Frequency (HF), and Verry Height Frequency (VHF).

The membership function linguist variables for the output $S_{k}(\mathrm{t})$ are: Negative Verry Height Priority (NVHP), Negative Height Priority (NHP), Negative Medium Priority (NMP), Negative Low Priority (NLP), Negative Verry Low Priority (NVLP), positive Verry Low Priority (PVLP), positive Low Priority (PLP), positive Medium Priority (PMP), positive Height Priority (PHP) and positive Verry Height Priority (PVH).

The fuzzy rules can be written as shown in Table 1. For this purpose, we used a system of Mamdani-type fuzzy logic and the method of center of gravity for Defuzzification of the output control. The output value represents the priority of the service to be executed. 

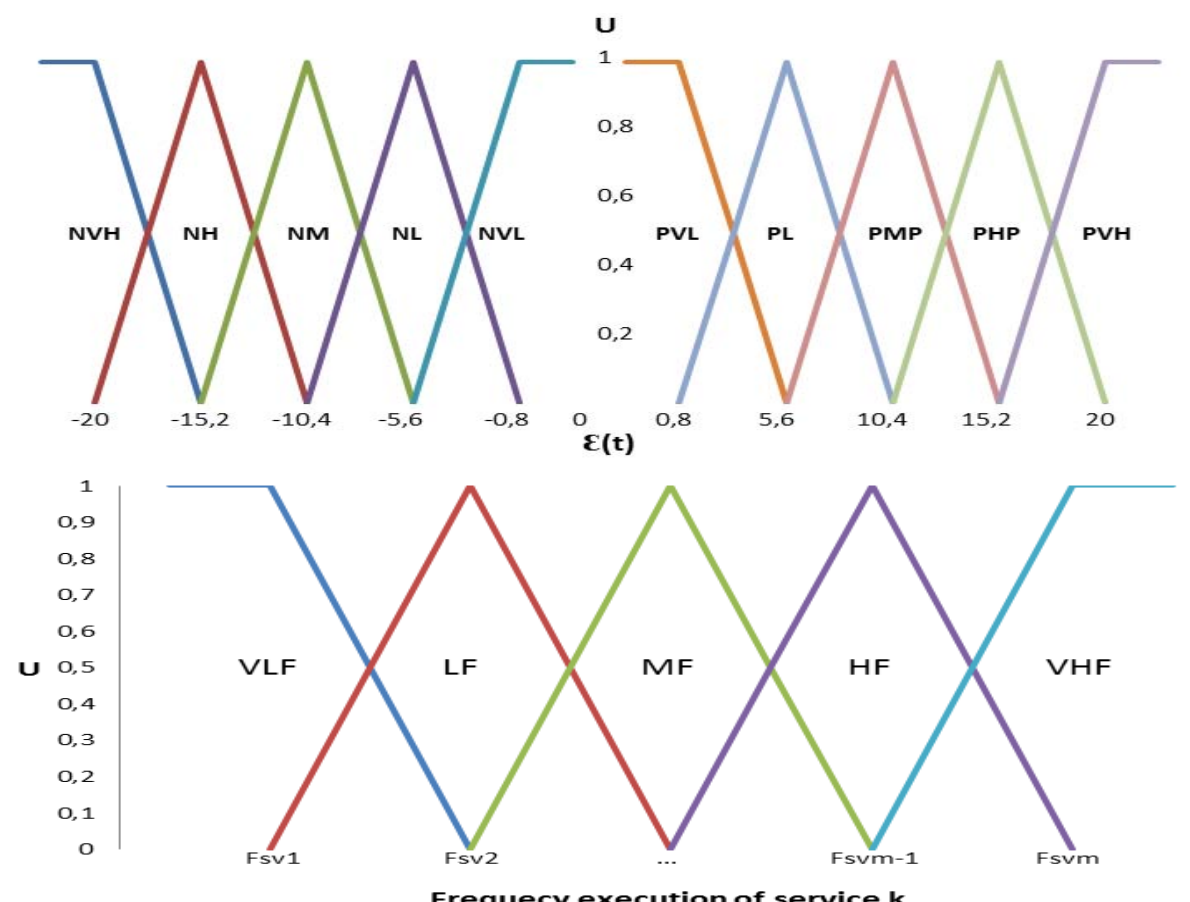

Frequecy execution of service $k$

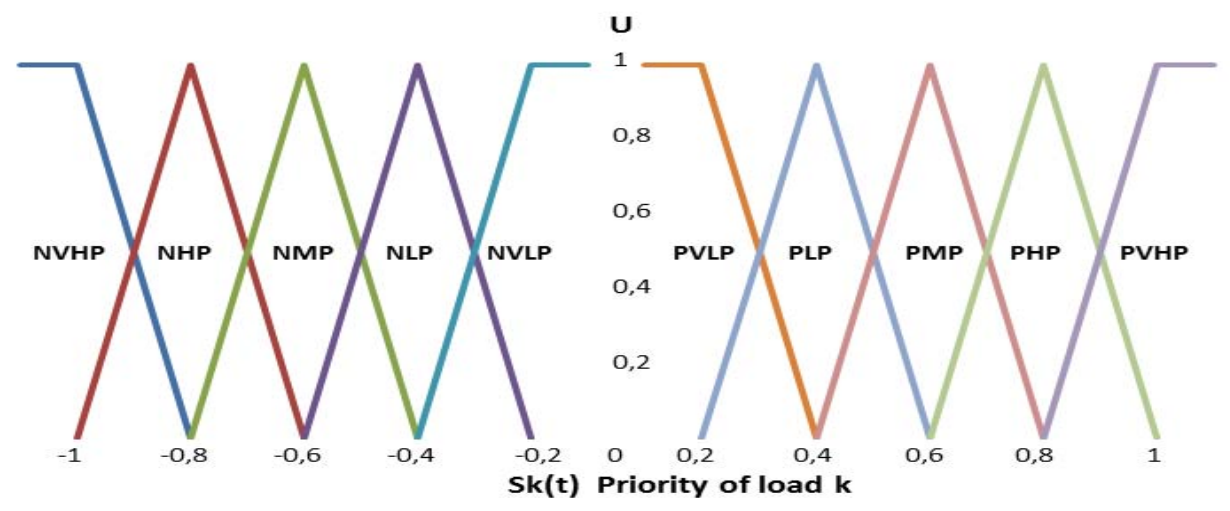

Fig 4. membership functions for Input and output of each FLCk

TABLE I Fuzzy rules for output (Sk(t)) of FLCk.

\begin{tabular}{|l|r|r|r|r|r|}
\hline \multirow{2}{*}{$\varepsilon(t)$} & \multicolumn{5}{|c|}{$F s v_{k}$} \\
\cline { 2 - 6 } & VL & \multicolumn{1}{|c|}{ L } & M & \multicolumn{1}{c|}{ H } & VH \\
\hline NVH & NVHP & NVHP & NVHP & NVHP & NVHP \\
\hline NH & NHP & NHP & NVHP & NVHP & NVHP \\
\hline NM & NMP & NHP & NHP & NVHP & NVHP \\
\hline NL & NLP & NMP & NMP & NHP & NVHP \\
\hline NVL & NVLP & NLP & NLP & NMP & NHP \\
\hline PVL & PL & PL & PVLP & PVLP & PVLP \\
\hline PL & PMP & PMP & PLP & PLP & PLP \\
\hline PM & PHP & PHP & PMP & PMP & PMP \\
\hline PH & PVHP & PVHP & PHP & PHP & PHP \\
\hline PVH & PVHP & PVHP & PVHP & PVHP & PVHP \\
\hline
\end{tabular}


C. The system demand management strategy

The demand management strategy is centered on the flexibility of loads. The flexibility allows the charge profile adaptation in relation with the system energy sources production. The general idea is to minimize the power deviation (Eq. (9)) between the renewable sources production and the load by modifying the charge profiles through its different local entities. This calls for the design and implementation of control strategies that could take into account the system dynamics which is characterized by unpredictable changes that require a permanent self-adjustment of the management system.

$$
\operatorname{Deviation}(t)=P_{W T}(t)+P_{P V}(t)+P_{B a t}-P_{L o a d}(t)
$$

With $P_{P V}(t)$ is the power supplied by the photovoltaic generator, $P_{W T}(t)$ that produced by the aerogenerator, $P_{B a t}(t)$ that produced or consumed by the battery. $P_{B a t}(t)$ is positive if the battery is a generating one and $P_{B a t}(t)$ is negative if the battery is a receiving one, $P_{\text {Load }}(t)$ is the energy called by the Load.

The Algorithm of the Energy coordination Control Strategy (ECCS) is described as follows:

\section{Algorithm ECCS}

Require initialization

While $(\mathrm{t}<=24)$

1. Calculate $P_{W T}(t), P_{P V}(t)$ and $P_{\text {load }}(t)$

2. Calculate $\mathrm{P}_{\mathrm{ren}}(t)=P_{\mathrm{WT}}(t)+\mathrm{P}_{\mathrm{PV}}(\mathrm{t})$ and $\varepsilon(t)=\mathrm{P}_{\mathrm{ren}}(t)-P_{\text {load }}(t)$

Calculate Deviation $(t)=\mathrm{P}_{\text {ren }}(t)+\mathrm{P}_{\text {Bat }}(\mathrm{t})-P_{\text {load }}(t)$

While $N<=N_{\max }$ do $\quad\left(N_{\max } \max\right.$ iteration)

3. If $(\varepsilon(t)=0)$ then

The generated power will be spent supplying the main load by WT and PV;

$$
N=N_{\max }
$$

4. Else if $(\varepsilon(t)>0)$ then (Excess generated power)

5. If $\left(\operatorname{soc}(t-1)<\operatorname{soc}_{\max }\right)$ then

Charge Battery equation 6;

$$
N=N_{\max } ;
$$

6.

$$
\text { Else }
$$

(deviation);

Deviation $(\mathrm{t})=\mathrm{LDk}$ Agent.FLCk.adaptation

$$
N++
$$

$7 . \quad$ End if

8. Else if $(\varepsilon(t)<0)$ then (Lack generated power)

9. If $\left(\operatorname{soc}(t-1)>\operatorname{soc}_{\text {min }}\right)$ then

Discharge Battery equation 7 and $N=N_{\max }$;

10. Else

(deviation);

$$
\text { Deviation }(\mathrm{t})=\mathrm{LDk} \text { Agent.FLCk.adaptation }
$$

$$
N++
$$

11. End if

\section{End if}

12. End While

Fig 5. Algorithm of the Energy Coordination Control Strategy (ECCS). 
D. Mathematical formulation of the control strategy

This section presents the mathematical formulation of the system management strategy. The control strategy is modeled mathematically by means of an optimization problem under constraints inspired by [11] work. The question is to minimize the Euclidean distance between production and consumption (Eq. (10)).

$$
F(t)=\sqrt{\sum_{t=1}^{N i}\left(\left(P_{\text {Load }}(t)-\operatorname{Pr} \text { oduction }(t)\right)^{2}\right.}
$$

With $P_{\text {Load }}(t)$ and Production $(t)$ are respectively the system's consumption and the production at instant $t$, Ni number of iteration. Production ( $\mathrm{t}$ ) is the power produced at instant $\mathrm{t}$ whose consumption $P_{\text {Load }}(t)$, given by Eq. (11), we want to extend.

$$
P_{\text {Lood }}(t)=\operatorname{Pr} \text { ediction }(t)+\text { connection }(t)-\text { Deconnection }(t)
$$

Where Prediction ( $(t)$ was the consumption predicted at instant $t$, Connection $(t)$ and Deconnection $(t)$ are respectively the quantities of energy consumed by the loads connected and disconnected at instant $t$ during the displacement in the temporary time space.

The minimization problem is subject to the following constraints (Equ. (12)): the amount of displaced loads is positive or zero. The amount of loads over a time interval cannot be superior to the equipment available for the control of the same interval.

$$
\begin{aligned}
& X_{k i t} \geq 0, \forall k, i, t \\
& X_{k i t} \leq \operatorname{Crlable}(i)
\end{aligned}
$$

With $X_{k i t}$ is the number of devices of type ${ }^{k}$ that are shifted from time step $i$ to $t$ and Ctrlable (i) is the amount of loads supplying the $\mathrm{k}$ type service available for control of instant $\mathrm{i}$.

\section{E. Process of energy coordination}

When the original power supply and demand balance of the power generation unit is broken, any Agent whose state changes can apply to be a Manager Agent to initiate the energy coordination task. The process of the coordination control algorithm is mainly including the following steps:

Step 1: release the bidding information.

Select Manager Agent (load balance) from the Agents applying for the energy coordination task. After obtaining the time-stamped Task Token, Manager Agent searches the current load and electricity price from LD Agent and sends the bid invitation to other Agents.

Step 2: agent enters a bid.

Each Agent organizes the tendering information based on the characteristics designed in Section 2.1 and submits the quad data $<\mathrm{B}, \mathrm{C}, \mathrm{S}>$ before the prescribed deadline.

Step 3: Task Agent evaluates the bid.

After receiving the bidding information, Manager Agent will make the energy coordination control with the ECCS algorithm stated in Section 3.A.

Step 4: find the bidding result.

Release the bidding result and the energy coordination control plan.

Step 5: Agent replies whether it has finished the task or not; if yes, the negotiation ends and Task Token fails; if not, re-initiate the energy coordination task.

\section{III.CASE STUDY}

This paper designs MAS with Java Agent Development (JADE) which is a FIPA (The Foundation for Intelligent Physical Agents) standard-based multi-Agent software development.

\section{A. Basic data}

The electrical system case studied consists of a $16.5 \mathrm{kWp}$ photovoltaic park made up of 110 module units of $150 \mathrm{~W}$ peach, a $20.5 \mathrm{~kW}$ Wind Park made up of 41 aerogenerator units of $500 \mathrm{~W}$ each and a $7200 \mathrm{~A}$ storage system made up of 160 battery units of $180 \mathrm{Ah}$ each and $12 \mathrm{~V}$.The $\mathrm{DC}$ bus voltage is $48 \mathrm{~V}$. The critical discharge level is $40 \%$. This allows the battery protection to be highly efficient. The simulations are carried out according to two scenarios. The first simulation scenario (S1) applies no control strategy. The second scenario (S2) applies the suggested control method. The two simulation results were then compared. 
The charges profiles are studied in this work (Fig. 6). The charge profile number1 corresponds to the use of public and commercial equipment (refrigerators, mills, pumping, radios, televisions, fans, computers, etc.). The demand total energy is $205 \mathrm{kWh} /$ day. The number 2 and 3 profile is characterized by a strong variation of the consumption.

Sex (6) service types provided by the loads have been considered (Table 2$)$ and one service a Svk $(k=1,2, \ldots, 6)$. the analysis of the charge profiles and the users behavior during the day make it possible to separate the hours of the day into 4 time intervals: I1 from 6 p.m. to 12 a.m. I2 from 14 a.m. to 6a.m, I3 from 6a.m. to 12 p.m. and I4 from 12 p.m. to 6 p.m.

TABLE III Types of service and the frequency execution in interval I of the loads.

\begin{tabular}{|l|l|l|l|l|l|l|}
\hline \multicolumn{1}{|c|}{ Services } & Sv1 & Sv2 & Sv3 & Sv4 & Sv5 & Sv6 \\
\hline$F_{S V k}$ & $6 / 6$ & $5 / 6$ & $4 / 6$ & $3 / 6$ & $2 / 6$ & $1 / 6$ \\
\hline
\end{tabular}

B. Results and analysis

Fig. 7 shows real-time illustration of the HES behavior, more particularly of the production evolution and the system consumption with and without ECCS application. The production is made up of the power supplied by the PV and the aerogenerator (Pren (t)) and the battery whereas the system consumption includes the power called by the loads and the battery charge power. Fig. 7 presents the results for profile 1 . Fig. 7 also shows an adaptation of the consumption in relation to the production: the consumption curve follows the production curve when the strategy is applied. The same trend is observed for profiles 2 and 3 and has made it possible to reduce the amount of energy lost when the ECCS was applied. The amount of energy lost has been evaluated with and without the application of the strategy. Figs. 8 and 9 show respectively the production and the consumption with and without the application strategy. They show a maximum deviation of $18 \mathrm{~kW}$ when the strategy is not applied and of $4 \mathrm{~kW}$ when the strategy is applied.

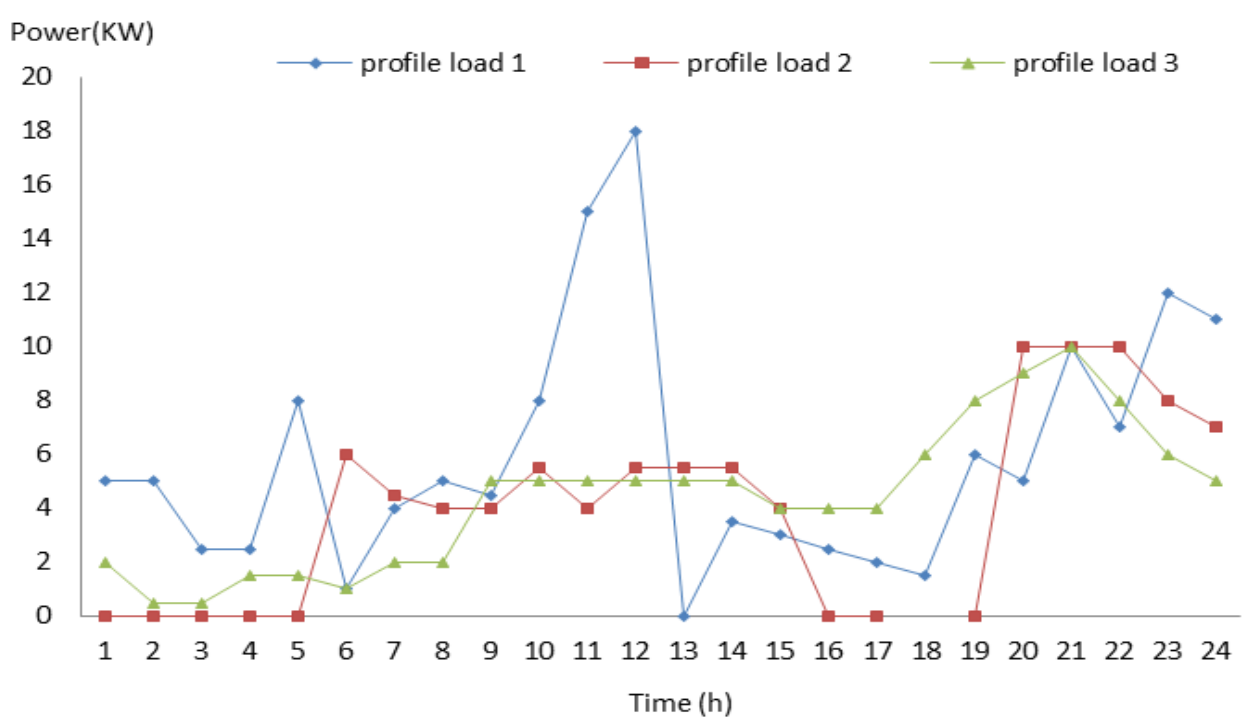

Fig 6. Three charge profiles of the loads.

Table 3 presents a comparison of the system performances with and without application of the suggested strategy. More than $31.13 \mathrm{kWh}$ of energy are lost every day with profile 1 when the strategy is not applied. The loss is evaluated to be higher than $22.627 \mathrm{kWh}$ a day with profile 2 and higher than $34.297 \mathrm{kWh}$ a day with profile 3. When the ECCS is applied, the losses are carried to less than $4.02 \mathrm{kWh}$ with profile 1, to about 3.438 $\mathrm{kWh}$ for profile 2 and to $4.219 \mathrm{kWh}$ for profile 3. Applying the proposed strategy has thus led to a gain of about $27.11 \mathrm{kWh}$ for profile $1,19.189 \mathrm{kWh}$ for profile 2 and $30.078 \mathrm{kWh}$ for profile 3 in one day (24h). From the economic point of view, the strategy applied on profile 1 has allowed gains superior to $2.711 \$$ a day that is more than $989.515 \$$ a year or more than 19790.3 \$ over the project life duration fixed for 20 years. The same trend is observed with profile 2 and 3. 


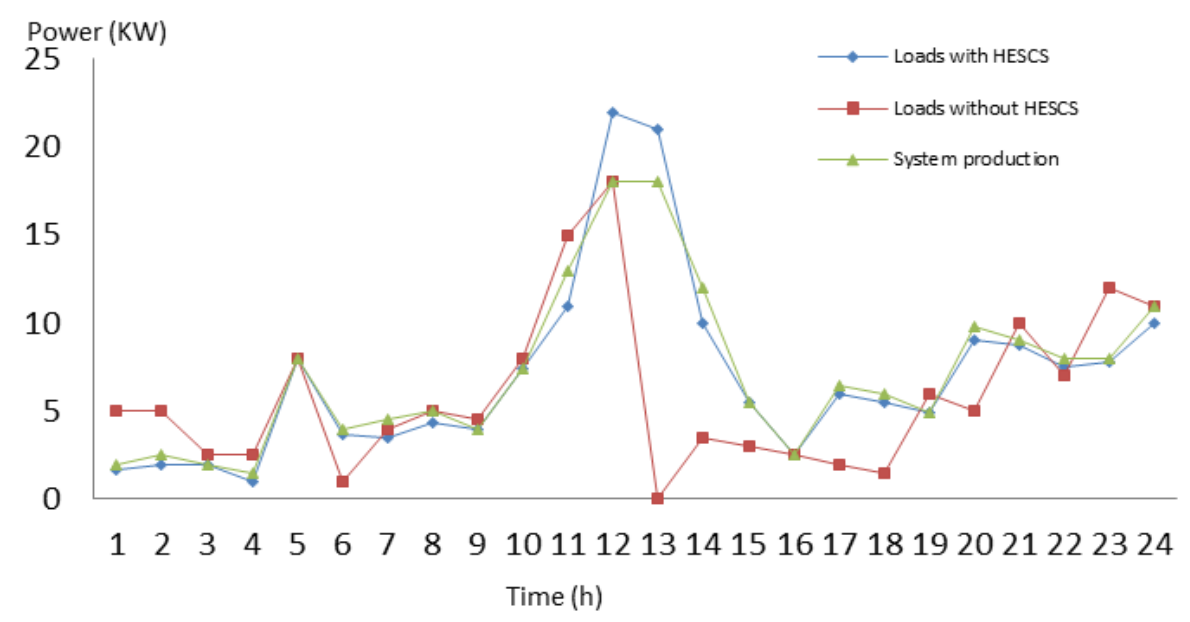

Fig 7. HES real-time function: with profile 1(without and with ECCS)

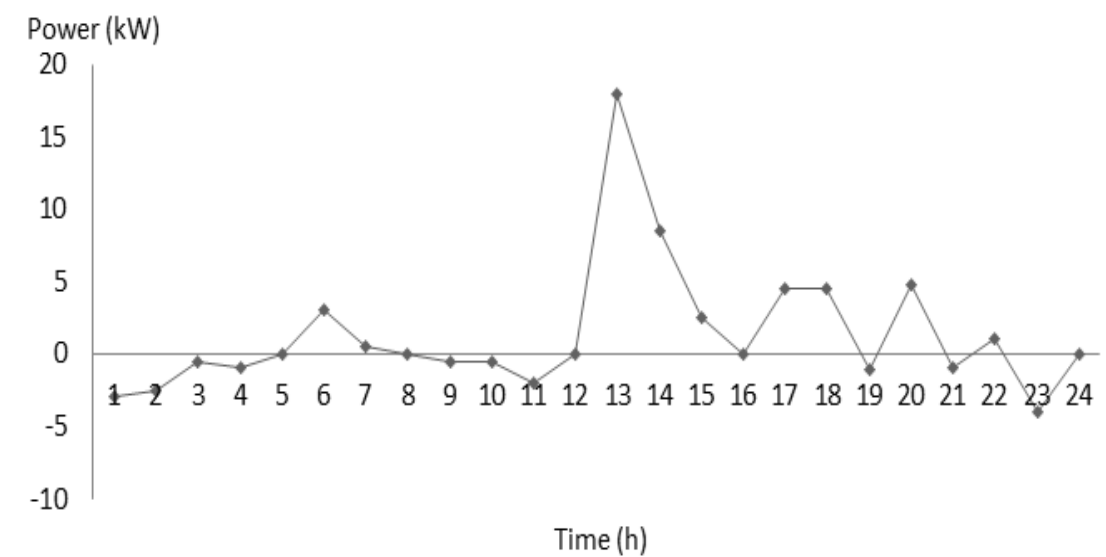

Fig 8.The power difference between the production and the consumption without application of the ECCS: profile 1.

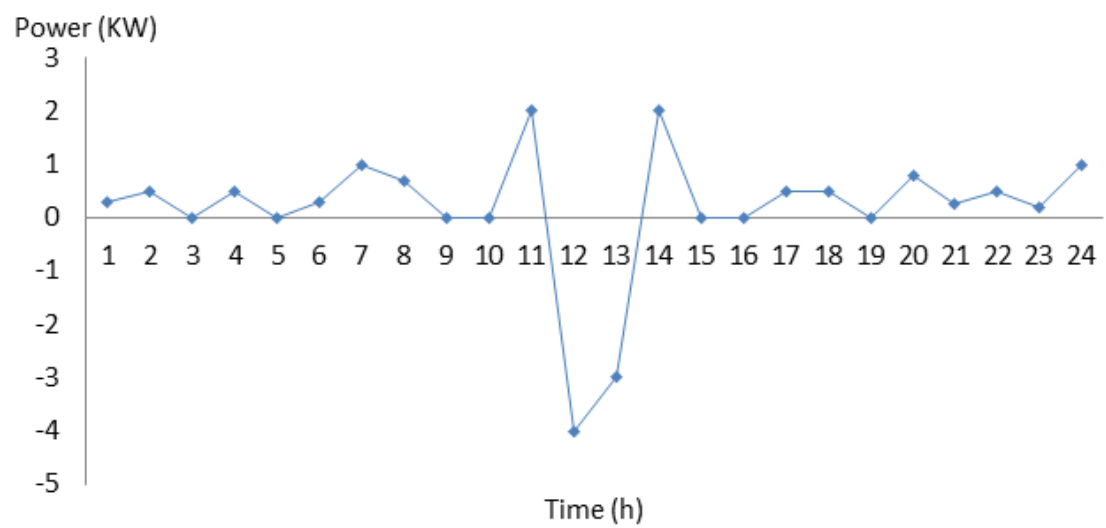

Fig 9.The power difference between the production and the consumption with application of the ECCS: profile 1.

TABLE IIIII Comparison the proposition energy and economic performance.

\begin{tabular}{|l|l|l|l|}
\hline & P1 & P2 & P3 \\
\hline Energy lost without application of ECCS & $31.13 \mathrm{KW}$ & $22.627 \mathrm{KW}$ & $34.297 \mathrm{KW}$ \\
\hline Energy lost with application of ECCS & $4.02 \mathrm{KW}$ & $3.438 \mathrm{KW}$ & $4.219 \mathrm{KW}$ \\
\hline Energy gained with application of ECCS & $27.11 \mathrm{KW}$ & $19.189 \mathrm{KW}$ & $30.078 \mathrm{KW}$ \\
\hline Cost of the energy gained in \$/day & 2.711 & 1.9189 & 3.0078 \\
\hline Cost of energy gained in \$/year & 989.515 & 700.3985 & 1097.847 \\
\hline
\end{tabular}




\section{IV.CONCLUSION}

The hybrid electrical system decentralized management strategy based on renewable energies is presented in this paper. The management strategy was implemented in a multi-agent architecture where different selfsufficient entities cooperated and controlled the energy flows exchanged among the system to reduce the energy produced by the renewable sources. They relied on the load control flexibility to reduce the gap between the production and the consumption. Using a situated model built around multi-agent paradigms was an innovative and promising option for the decentralized control of system. The methodology is based on the possibility of shifting, changing and interrupting the service demand used. The control strategy developed was applied on a solar, wind, battery hybrid electrical system and three charge profiles were considered in the simulations. The simulation results showed that the consumption was adapted to the production: the consumption curve followed the production curve when the strategy is applied on the charge profiles. The control strategy also made it possible in one day (24h) to gain about $27.11 \mathrm{KWh}$ for profile1, $19.189 \mathrm{KWh}$ for profile 2 and $30.078 \mathrm{KWh}$ for profile 3. From an economic point of view the strategy applied on profile 1 allowed gains superior to $2.711 \$$ a day that is more than $989.515 \$$ per year or more than $19790.3 \$$ over a period of 20 years, the time decided up on as the project life duration. The same trend was observed with profiles 2 and 3.

\section{REFERENCES}

[1] Herna Sndez L, Baladron C, Aguiar JM, Carro B. A multi-agent system archi tecture for smart grid management and forecasting of energy demand in virtual power plants. In: Proceedings of the IEEE communications magazine, Vol. 51.Issue1; 2013.p.106-13.

[2] Thillainathan L, Ashwin MK. Multiagent system for real-time operation of a microgrid in real-time digital simulator. IEEE Trans Smart Grid 2012; 3: 2.

[3] Sofla MA, King R. Control method for multi-microgrid systems in smart grid environment stability,optimization and smart demand participation. In: Proceedings of the innovative smart grid technologies (ISGT); 2012. p. 1-5.

[4] Aung HN, Khambad kone AM, Srinivasan D, LogenthiranT. Agent-based intelligent control for real-time operation of a microgrid Joint. In: Proceedings of the international conference on power electronics drives and energy systems; 2010.p.1-6.

[5] Dimeas AL, Hatziargyriou ND. Multi-agent reinforcement learning for micro- grids. In: Proceedings of the IEEEPES. General meeting; 2010p.1-8.

[6] LiT, Xiao Z, Huang M, Yu J, Hu J. Control system simulation of microgrid based on IP and Multi Agent. In: Proceedings of the international conference on information, networking and automation (ICINA); 2010.p.V1-235-39.

[7] Ng EJ, El-Shatshat RA. Multi-microgrid control systems (MMCS). In:Proceedings of the IEEEPES. Generalmeetingn; 2010.p.1-6.

[8] Phillips L, Link H,Smith R,Weiland L. Agent-based control of distributed infrastructure resources. Sandia National Laboratories, SAND 2005-7937; 2006. Available: www.sandia.gov/scada/documents/sand_2005_7937.pdf.

[9] Rocabert J, Azevedo G, Vazquez G, CandelaI, Rodriguez P, Guerrero J. M. Intelligent control agent for transient to an island grid. In: Proceedings of the IEEE international symposiumo $n$ industrial electronics; 2010.p.2223-28.

[10] Zheng G. LiN. Multi-Agent Based Control System for Multi-Microgrids. In: Proceedings of the international conference on computational intelligence and software engineering; 2010.p.1-4.

[11] Zheng W, Cai J. A multi-agent system for distributed energy resources control in microgrid. In:Proceedings of the 5th international conference on critical infrastructure (CRIS); 2010.p.1-5.

[12] M bodji A K, Ndiaye ML,Ndiaye PA. Decentralized management of a multi-source electrical system: A multi-agent approach. Acad J 2013; 8(22): 1256-65 ISSN 1992-1950.

[13] Monica A, Hortensia A, Carlos AO. Integration of renewable energy sources in smart grids by means of evolutionary optimization algorithms. Expert Syst Appl 2011; 39:5513-22.

[14] Mao M, Dong W, Liuchen C. Design of a novel simulation plat form for the EMS-MG based on MAS. In: Proceedings of the IEEE energy conversion congress and expositionenergy conversion innovation for a clean energy; 2011.p.2670-75.

[15] Pipattanasomp M, Feroze H, Rahman S. Multi-Agent Systems in a Distributed Smart Grid: Design and Implementation. in: Proceedings of the IEEEPES power systems conference and exposition (PSCE'09), Seattle, Washington, USA; 2009.

[16] Logenthiran T, Srinivasan D, Khambadkone A M, Aung HN. Multi agent system for real-time operation of a microgrid in real-time digital simulator. IEEE Trans Smart Grid 2012 ;3(2): 925-33.

[17] Kazem, H.A., Khatib, T., 2013. A novel numerical algorithm for optimal sizing of a photovoltaic/wind/diesel generator/battery microgrid using loss of load probability index. Int. J. Photoenergy.

[18] Yang, H., Wei, Z., Chengzhi, L., 2009. Optimal design and technoeconomic analysis of a hybrid solar-wind power generation system. Appl. Energy 86 (2), 163-169.

[19] Yang, H., Lu, L., Zhou, W., 2007. A novel optimization sizing model for hybrid solar-wind power generation system. Sol. Energy 81 (1), 76-84.

\section{AUTHOR PROFILE}

Author1 Name Zaid BARI, Laboratory of Sciences of the Engineer (LSI), Polydisciplinary Faculty of Taza, BP. 1223, Taza, Sidi Mohamed Ben Abdellah University, Fez, Morocco.

Author2 Name Majid BEN YAKHLEF, Laboratory of Sciences of the Engineer (LSI), Polydisciplinary Faculty of Taza, BP. 1223, Taza, Sidi Mohamed Ben Abdellah University, Fez, Morocco. 